\title{
Development of the nano-HEB array for low-background far-IR applications
}

\author{
Boris S. Karasik*a, Sergey V. Pereverzeva ${ }^{\mathrm{a}}$, David Olaya ${ }^{\mathrm{b}}$, Michael E. Gershenson ${ }^{\mathrm{c}}$, Robin Cantor ${ }^{\mathrm{d}}$, \\ Jonathan H. Kawamura ${ }^{a}$, Peter K. Day ${ }^{a}$, Bruce Bumble ${ }^{a}$, Henry G. LeDuc ${ }^{a}$, Steve P. Monacos ${ }^{a}$, \\ Dennis G. Harding ${ }^{\mathrm{a}}$, Daniel Santavicca ${ }^{\mathrm{e}}$, Faustin Carter $^{\mathrm{e}}$, and Daniel E. Prober ${ }^{\mathrm{e}}$ \\ a Jet Propulsion Laboratory, California Institute of Technology, \\ 4800 Oak Grove Dr., Pasadena, CA 91109-8001; \\ ${ }^{b}$ National Institute of Standards and Technology, 325 Broadway, Boulder, CO 80305-3328; \\ ${ }^{c}$ Rutgers University, 136 Frelinghuysen Rd., Piscataway, NJ 08854-8019; \\ ${ }^{\mathrm{d}}$ STAR Cryoelectronics, 25-A Bisbee Court, Santa Fe, NM 87508-1412; \\ 'Yale University, 15 Prospect St, New Haven, CT 06511-6816
}

\begin{abstract}
We present an overview of the recent progress made in the development of a far-IR array of ultrasensitive hot-electron nanobolometers (nano-HEB) made from thin titanium (Ti) films. We studied electrical noise, signal and noise bandwidth, single-photon detection, optical noise equivalent power (NEP), and a microwave SQUID (MSQUID) based frequency domain multiplexing (FDM) scheme. The obtained results demonstrate the very low electrical $N E P$ down to $1.5 \times 10^{-20} \mathrm{~W} / \mathrm{Hz}^{1 / 2}$ at $50 \mathrm{mK}$ determined by the dominating phonon noise. The $N E P$ increases with temperature as $\sim T^{3}$ reaching $\sim 10^{-17} \mathrm{~W} / \mathrm{Hz}^{1 / 2}$ at the device critical temperature $T_{C}=330-360 \mathrm{mK}$. Optical $N E P=8.6 \times 10^{-18} \mathrm{~W} / \mathrm{Hz}^{1 / 2}$ at 357 $\mathrm{mK}$ and $1.4 \times 10^{-18} \mathrm{~W} / \mathrm{Hz}^{1 / 2}$ at $100 \mathrm{mK}$ respectively, agree with thermal and electrical data. The optical coupling efficiency provided by a planar antenna was greater than $50 \%$. Single $8-\mu \mathrm{m}$ photons have been detected for the first time using a nano-HEB operating at 50-200 $\mathrm{mK}$ thus demonstrating a potential of these detectors for future photon-counting applications in mid-IR and far-IR. In order to accommodate the relatively high detector speed $(\sim \mu \mathrm{s}$ at $300 \mathrm{mK}, \sim 100 \mu \mathrm{s}$ at $100 \mathrm{mK}$ ), an MSQUID based FDM multiplexed readout with $\mathrm{GHz}$ carrier frequencies has been built. Both the readout noise $\sim 2 \mathrm{pA} / \mathrm{Hz}^{1 / 2}$ and the bandwidth $>150 \mathrm{kHz}$ are suitable for nano-HEB detectors.
\end{abstract}

Keywords: hot-electron, nanobolometer, single-photon, MSQUID, FIR detector, antenna-coupled

\section{INTRODUCTION}

Recently, we demonstrated and measured extremely low thermal conductance in Ti nano-HEBs due to the weak electron-phonon interaction in a small device volume of about $10^{-21}-10^{-20} \mathrm{~m}^{3}$ [1]. The electron-phonon thermal conductance, $G_{e-p h}$, has stronger temperature dependence than that of phonon transport in narrow $\mathrm{Si}_{3} \mathrm{~N}_{4}$ beams commonly used in geometrically isolated bolometers [2]. In fact, $G_{\text {e-ph }}$ is about two orders of magnitude smaller at $40-50 \mathrm{mK}$ than the lowest thermal conductance found in suspended bolometers [3]. The very low $G_{e-p h} \approx 0.1 \mathrm{fW} / \mathrm{K}$ at $40 \mathrm{mK}$ found in [1] suggests the very low Thermal Energy Fluctuation (TEF) (aka phonon) noise $N E P_{T E F} \approx 3 \times 10^{-21} \mathrm{~W} / \mathrm{Hz}^{1 / 2}$ which matches to the photon shot noise expected for the cosmic background in the most sensitive instruments for moderate resolution submillimeter spectroscopy in space [4-6].

It turns out that the low electron heat capacitance $C_{e}=10^{-19}-10^{-18} \mathrm{~J} / \mathrm{K}$ in nano-HEB makes detection of single mid-IR and FIR photons feasible [1,7]. Calorimetry using transition-edge sensors (TES) is common in X-ray range and even at optical wavelengths but has not been achieved yet at long IR wavelengths. The possible applications include THz photon counting detectors for moderate resolution spectroscopy in space [7], low-resolution spectroscopy in mid-IR for Earthlike exoplanet search, and single molecule IR spectroscopy.

* boris.s.karasik@jpl.nasa.gov; phone 1818 393-4438; fax 1818 393-4683 
In our recent work [2], we studied the electrical noise of small nano-HEBs (volume $10^{-20}-10^{-19} \mathrm{~m}^{3}$ ) and found it being mostly dominated by the TEF noise. Electrical $N E P_{\text {TEF }}$ derived from those measurements was as low as $\sim 10^{-18} \mathrm{~W} / \mathrm{Hz}^{1 / 2}$ for smallest devices at $350 \mathrm{mK}$. Using a novel microwave technique [8] based on generation of short 20-GHz pulses imitating THz photons, we measured the energy resolution in similar nano-HEBs and found that $\Delta \varepsilon_{F W H M} / h \approx 23 \mathrm{THz}$ is feasible at $300 \mathrm{mK}$.

In the current work, we carried out a more detailed study of the nano-HEB electrical noise and the bandwidth as function of the operating point position and temperature. From these measurements, electrical $N E P$ as low as $1.5 \times 10^{-20} \mathrm{~W} / \mathrm{Hz}^{1 / 2}$ was obtained at $50 \mathrm{mK}$. We have observed a strong modification of the thermal time constant (both increase and decrease) due to the interplay of the positive and negative electro-thermal (ETF) feedback. Using a cryogenic blackbody source with a bandpass filter we performed direct optical NEP measurements with an antenna-coupled nano-HEB device and found good agreement between optical and electrical $N E P_{T E F}$ data indicating high optical coupling efficiency of the $650 \mathrm{GHz}$ planar antenna. When the very low optical $N E P \sim 10^{-18} \mathrm{~W} / \mathrm{Hz}^{1 / 2}$ was realized, we observed the fluctuations of radiation power (photon shot noise). We also studied the single photon detection effects in nano-HEBs employing a pulsed $8-\mu \mathrm{m}$ quantum cascade laser (OCL) as photon source. For the first time, very long-wavelength 8- $\mu \mathrm{m}$ single photons with some energy resolution of $\sim 1.4$ were detected using a bolometer. Finally, the ongoing work develops the MSQUID readout for nano-HEBs using a fully digital FDM scheme.

\section{DEVICES AND EXPERIMENTAL SETUP}

Parameters of the devices used in the work are listed in Table 1. Device \#1 was fabricated using a double-angle e-beam evaporation of $\mathrm{Ti}$ and $\mathrm{Nb}$ though an organic resist mask (see [1] for fabrication details). Devices \#2 and \#3 were fabricated using magnetron sputtering of Ti target and were patterned using optical lithography. NbTiN/Au bi-layer was used as the contact material for antenna-coupled devices \#2.

Table 1. Parameters of Ti nano-HEBs.

\begin{tabular}{|c|c|c|c|c|c|c|c|}
\hline Device & Substrate & $\begin{array}{c}\text { Length } \\
(\mu \mathrm{m})\end{array}$ & $\begin{array}{c}\text { Width } \\
(\mu \mathrm{m})\end{array}$ & $\begin{array}{c}\text { Thickness } \\
(\mathrm{nm})\end{array}$ & $\begin{array}{c}T_{C} \\
(\mathrm{mK})\end{array}$ & $\begin{array}{c}R_{N} \\
(\Omega)\end{array}$ & $\begin{array}{c}G_{e-p h} @ T_{C} \\
(\mathrm{pW} / \mathrm{K})\end{array}$ \\
\hline 1 & $\mathrm{Si}$ & 6.0 & 0.4 & 56 & 330 & 55 & 7 \\
\hline 2 & sapphire & 2.0 & 1.0 & 20 & 360 & 51 & 6 \\
\hline 3 & sapphire & 20 & 1.0 & 40 & 480 & 120 & - \\
\hline
\end{tabular}

$R_{N}$ is the device normal resistance; $G_{e-p h}$ is the thermal conductance due to the electron-phonon coupling in Ti film.

Noise, $N E P$, and single-photon detection measurements were performed in a dilution refrigerator. The cryogenic setup allowed for reconfiguration of radiation sources while using the same bias and readout lines. The devices were biased via a $1-\Omega$ resistor connected in series with the device and with the SQUID input coil. The resistor was situated in the vicinity of the device on the mixing chamber. The SQUID amplifier was placed at the $1-\mathrm{K}$ pot of the dilution refrigerator and was connected to the device circuit via a magnetically shielded superconducting twisted pair. The device assembly was in an rf tight superconducting shield in order to avoid overheating of the device by uncontrolled rf interferences and noise. The bias lines and the SQUID wires were fed through custom low-pass filters (LPF), which were built from discrete element LPFs placed inside metal tubes filled with lossy microwave absorbing compound. The SQUID noise ( $\left.2 \mathrm{pA} / \mathrm{Hz}^{1 / 2}\right)$ and bandwidth $(\sim 70 \mathrm{kHz})$ were calibrated in a separate experiment using the Johnson noise of a 1- $\Omega$ resistor at different temperatures.

In order to perform measurements at different temperatures below $T_{C}$ a small superconducting solenoid with an iron core was mounted near the bolometer. The perpendicular field created by the solenoid was enough to suppress $T_{C}$ down to 25 $\mathrm{mK}$ with the solenoid current not exceeding $200 \mathrm{~mA}$. The entire experimental volume was surrounded by a Cryoperm- $10^{\mathbb{R}}$ magnetic shield immersed in liquid He4.

Time constant and single-photon detection were studied using pulsed quantum cascade lasers (QCL) with $\lambda=100 \mu \mathrm{m}$ and $\lambda=8 \mu \mathrm{m}$ placed at $4 \mathrm{~K}$. The lasers shared the same vacuum with the nanobolometer devices. The radiation was delivered to the nano-HEB via a lossy lightpipe through a $10 \%$ bandpass mesh filter centered at $\lambda=100 \mu \mathrm{m}$ and small apertures at still and mixing chamber temperatures. The entire transmission was adjusted to attenuate the number of photons per pulse to less than 1 so the Poisson count statistics could be realized. 
Optical NEP was measured using a black body source made from a metal disc in which a thermometer and a small heater were embedded. The emitting surface was machined into an array of small pyramids and was painted with an FIR absorbing paint whose high (97\%) absorptivity was validated by both direct measurements of the reflection at around $600 \mathrm{GHz}$ and comparison with the emissivity of foamed Eccosorb used as the standard calibration target for a heterodyne receiver. This black body assembly was weakly coupled to the $1 \mathrm{~K}$ pot so its temperature could be controlled with a better than $1 \%$ precision from $1.5 \mathrm{~K}$ to $10 \mathrm{~K}$.

\section{EXPERIMENTAL RESULTS}

\subsection{Electrical noise and bandwidth}

Device \#1 was used in these measurements. Figure 1 shows a family of the current-voltage characteristics (IVC) for this device in the vicinity of the critical temperature offset by a few $\mathrm{mK}$ from each other. From this data, the thermal conductance due to the intrinsic electron-phonon coupling was determined (Table 1, see [2,9] for details of the technique). The corresponding $N E P_{T E F}=\sqrt{4 k_{B} G_{e-p h} T_{C}^{2}}=6.5 \times 10^{-18} \mathrm{~W} / \mathrm{Hz}^{1 / 2}$ does not depends on the bias point as long as the TEF noise dominates.

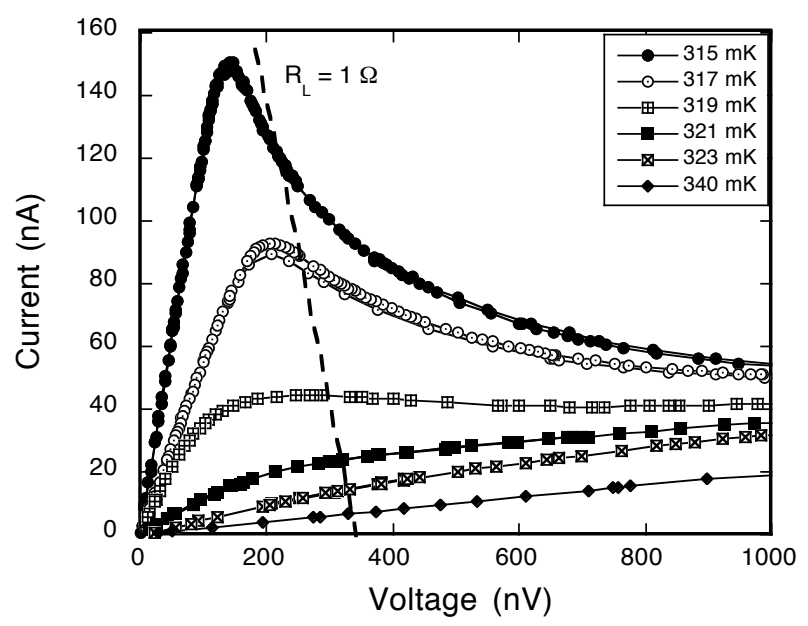

Figure 1. IVCs vs temperature for device \#1 near $T_{C}$.

Experimental noise spectra at $315 \mathrm{mK}$ are shown in Fig. 2. The curves denoted with letters (a)-(d) refer to the bias voltages denoted with the same letters in Fig. 3. That is, noise level is the highest at the bias point just beyond the top of the IVC and gradually decreases as the bias voltage increases. The output noise well exceeds the SQUID noise $i_{n}^{S Q U I D} \approx$ $2.5 \mathrm{pA} / \mathrm{Hz}^{1 / 2}$, which dominates in the normal state.

Even though the cut-off frequency $f_{-3 d B}$ of the noise spectra were close to the SQUID cut-off of $70 \mathrm{kHz}$, its dependence on the bias point was clearly observed. The corresponding time constant $\tau^{*}=(2 \pi f-3 d B)^{-1}$ is plotted in Fig. 3 along with the prediction of the bolometric model of [10] taking in account the ETF mechanism in the most general form:

$$
\tau^{*}=\tau \frac{d V / d I+R}{2 R} \frac{R+R_{L}}{d V / d I+R_{L}}
$$

Here $\tau=C_{e} / G_{e-p h}$ is the physical time constant which is observed when the ETF is absent (e.g., a very small bias current is used when the device is near $\left.T_{C}\right)$. When $R_{L}=0$, Eq. 1 coverts into a more familiar expression $\tau^{*}=\tau /(1+L)$ that corresponds to the strong negative ETF. $L=(d V / d I-R) /(d V / d I+R)$ is the ETF loop gain. In the following, $R_{L}=1.8 \Omega$ is used reflecting the fact than beside a $1-\Omega$ bias resistor, an additional $0.8-\Omega$ residual resistance was in series with the device in the ETF loop. 


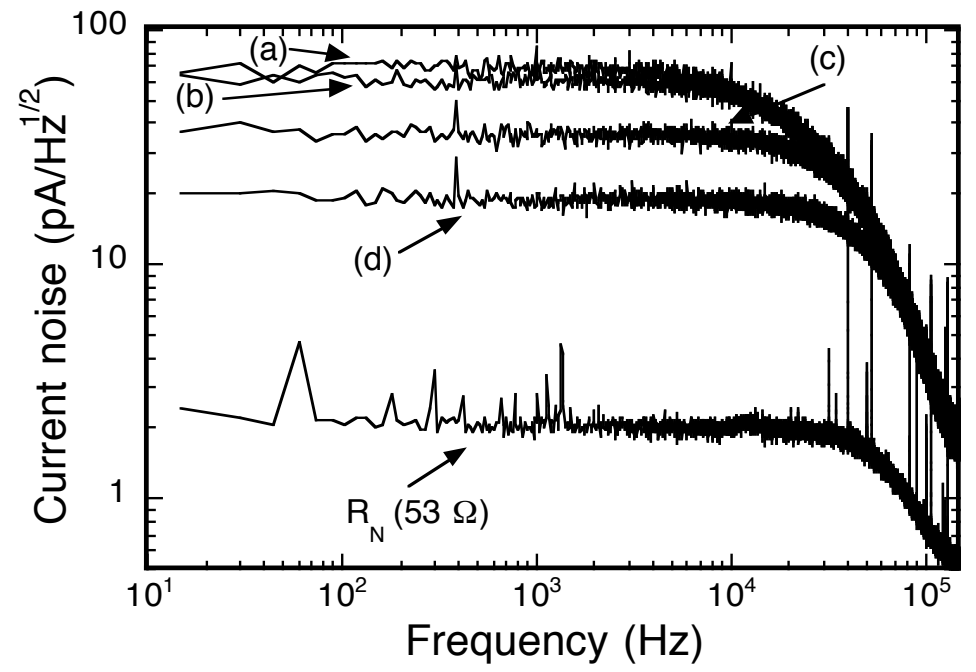

Figure 2. Output noise spectra for device \#1 as function of the bias point. The positions of bias points (a)-(d) are shown in Fig. 3.

According to Eq. 1, the time constant should decrease sharply with the bias voltage above $150 \mathrm{nV}$ (see Fig. 3) that corresponds to the negative ETF. However, a saturation is observed at $\tau^{*} \approx 3 \mu$ s because of the SQUID bandwidth limit. An inset in Fig. 3 shows what would happen if $R_{L}$ were smaller than $1.8 \Omega$. In this case, the transition from the positive $\operatorname{ETF}\left(\tau^{*} / \tau>1\right)$ to the negative $\operatorname{ETF}\left(\tau^{*} / \tau<1\right)$ could occur at smaller bias voltages, that is, mostly a negative ETF would take place.

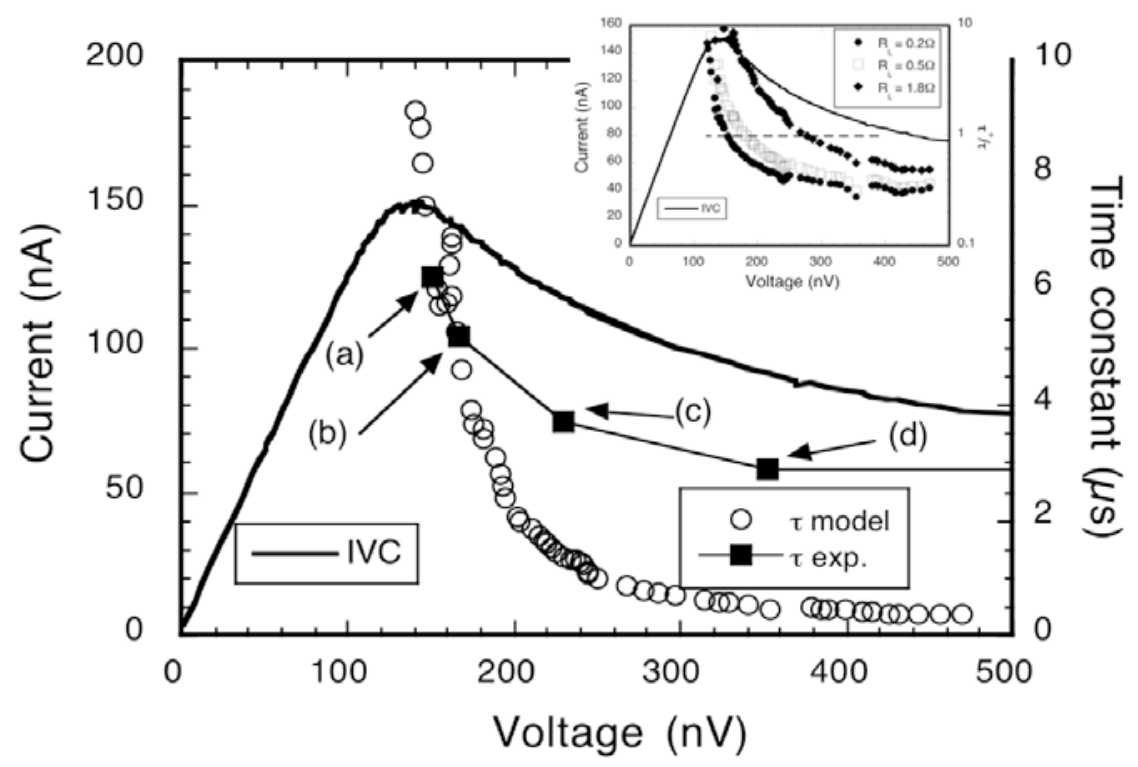

Figure 3. IVC and time constant for device \#1 at $315 \mathrm{mK}$. The time constant data points (a)-(d) are determined from the cutoff frequencies of the spectra in Fig. 2. The inset shows the ETF effect on the time constant for different load resistors.

The magnitude of the noise $i_{n}$ is well described by the expression $i_{n}=N E P_{T E F} \cdot S_{I}$, where

$$
S_{I}=\frac{\Delta I}{P_{\text {rad }}}=\frac{1}{2 I R_{L}} \frac{(d V / d I) / R-1}{(d V / d I) / R_{L}+1}
$$


is the detector current responsivity to the incident radiation power $P_{\text {rad }}$ [10]. In the strong negative ETF limit, Eq. 2 simplifies and $S_{I}=(1 / V) L /(L+1)$. Such behavior of the output detector noise confirms its TEF nature. The Johnson noise contribution was negligible under conditions of our experiment.

A similar behavior of the noise spectra was observed at lower temperatures where $T_{C}$ was lowered using a magnetic field. Figure 4 shows an example of the noise spectra measured in different bias points at $150 \mathrm{mK}$ shown in Fig. 5. Here $\tau=100 \mu$ s so the $70 \mathrm{kHz}$ readout bandwidth span was sufficient to trace to crossover between the ETF noise and the SQUID noise. Point (a) corresponds to the positive ETF, so $\tau^{*} / \tau \gg>1$ and the ETF noise cut-off occurs at $\sim 300 \mathrm{~Hz}$ but the $N E P_{T E F}$ remains constant up to $\sim 10 \mathrm{kHz}$ where $i_{n}^{S Q U I D}$ begins to dominate. Point (b) corresponds to the situation when the ETF did not affect $\tau^{*}$ so the cut-off of the TEF spectrum corresponds to $(2 \pi \tau)^{-1}$.

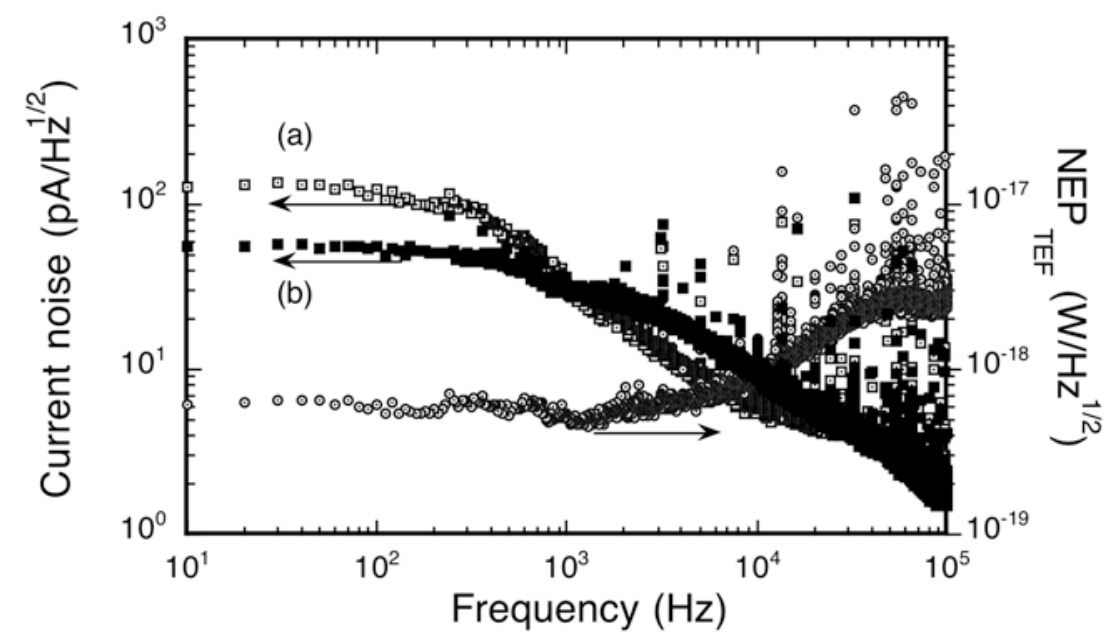

Figure 4. Noise spectra and $N E P_{T E F}$ for device $\# 1$ at $150 \mathrm{mK}$. Spectral curves denoted as (a) and (b) correspond to the bias point in Fig. 5 denoted with the same letters.

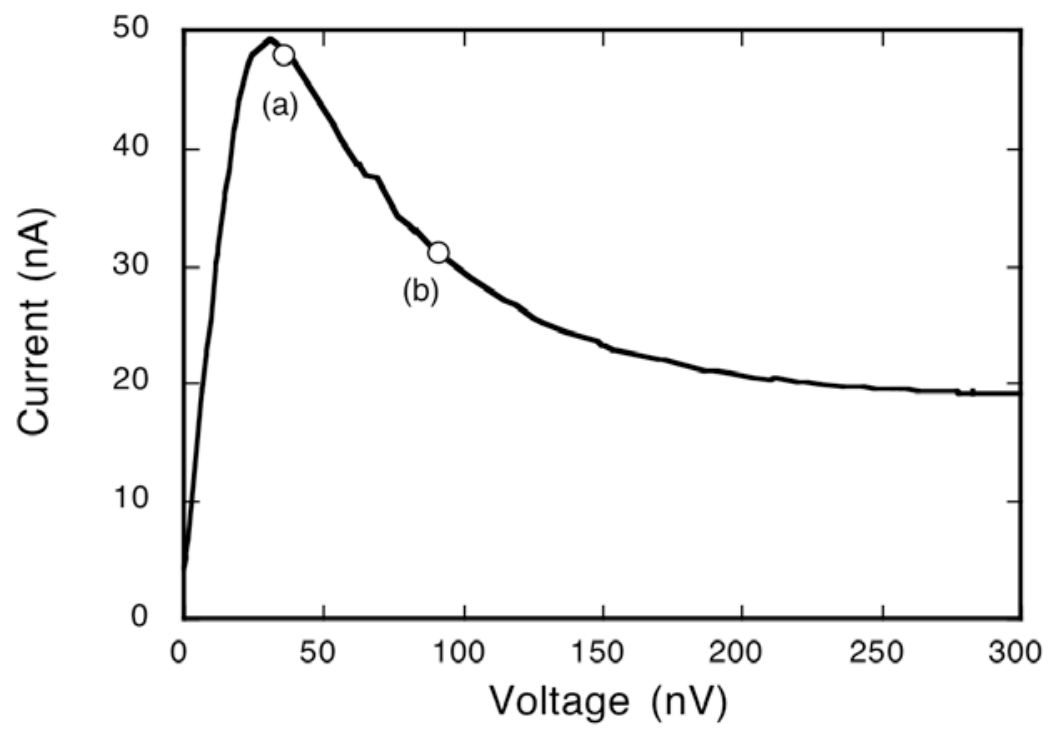

Figure 5. IVC for device $\# 1$ at $150 \mathrm{mK}$.

Figure 6 shows a summary of the $N E P_{T E F}$ as function of temperature along with the physical time constant measured using a short pulse from the QCL laser and a very small bias current. Between $150 \mathrm{mK}$ and $300 \mathrm{mK}, \tau(T) \sim T^{4}$ which agrees with the theory [11] prediction. The temperature dependence weakens below $100 \mathrm{mK}$ that is likely to some uncontrolled overheating of the electron subsystem by the spurious rf noise from the SQUID or through bias lines, or 
from the 4-K thermal emission through the laser light pipe. Nevertheless, the very low $N E P_{T E F}=1.5 \times 10^{-20} \mathrm{~W} / \mathrm{Hz}^{1 / 2}$ has been measured at $50 \mathrm{mK}$. The corresponding $G_{e-p h}=1.6 \mathrm{fW} / \mathrm{K}$ so the spurious signal power as low as $0.01 \mathrm{fW}$ could make a noticeable effect on the electron temperature.

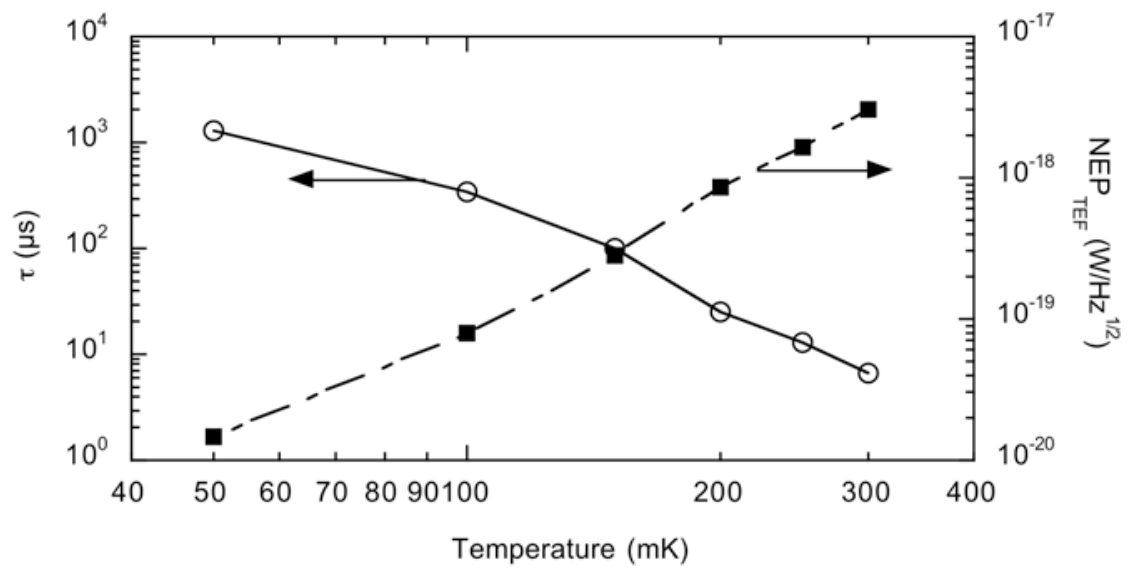

Figure 6. Physical time constant $\tau$ and $N E P_{T E F}$ as function of temperature for device $\# 1$.

\subsection{Optical NEP}

Optical NEP was measured using device \#2 fabricated along with the twin-slot planar antenna and a coplanar waveguide on the same chip. The chip was glued to the backside of a $12-\mathrm{mm}$ diameter Si lens thus forming a hybrid antenna (Fig. 7) with a well-defined and narrow diffraction limited beam. The antenna bandwidth was centered at $650 \mathrm{GHz}$. This frequency was sufficiently low to allow for definition of all the narrow gaps and slot in the layout by optical lithography with sufficient precision. This design was introduced in [12] and since then has been successfully used in a number of submillimeter heterodyne receivers including Herschel HIFI THz HEB mixers.
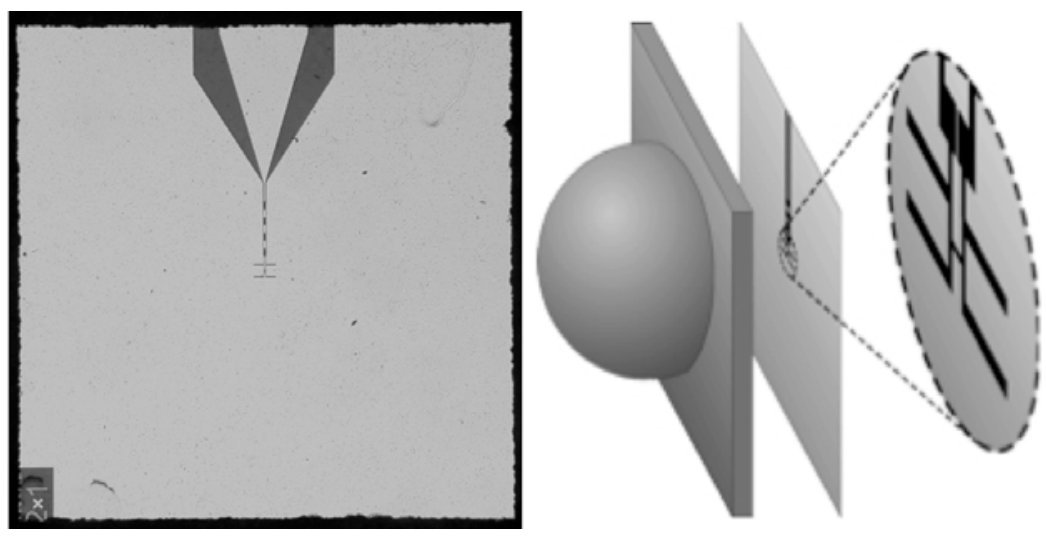

Figure 7. Device \#2 chip layout and its attachment to the lens. The chip size is $5 \mathrm{~mm} \times 5 \mathrm{~mm}$. The lens diameter is $12 \mathrm{~mm}$ and is out of scale on this figure.

In order to define the optical bandwidth a mesh filter also centered at $650 \mathrm{GHz}$ was used. The filter transmission spectrum $\operatorname{Tr}(v)$ was previously characterized using an FTS. Since our hybrid antenna couples only to a single radiation mode the amount of radiation power incident on the detector can be calculated as follows:

$$
P_{r a d}=\int_{0}^{\infty} \frac{\operatorname{Tr}(v) h v d v}{\exp \left(h v / k_{B} T_{B B}\right)-1}
$$

The black body temperature range $T_{B B}=1.5-5 \mathrm{~K}$ was sufficient to observe the entire evolution of the detector output signal and noise up to the full saturation of the output $\left(R \approx R_{N}\right)$. Figure 8 shows dependencies of the bias current decrease 
$\Delta I$ and the output noise for two device temperatures. At $357 \mathrm{mK}$, the nano-HEB was close to $T_{C}$ and no magnetic field was used. From the slope of initial part of $\Delta I$ vs $P_{\text {rad }}$ curve, the responsivity $S_{I}=\Delta I / P_{\text {rad }}$ was determined and the "dark" optical $N E P$ was estimated as $i_{n}\left(P_{r a d}=0\right) / S_{I}=8.6 \times 10^{-18} \mathrm{~W} / \mathrm{Hz}^{1 / 2}$. The same procedure gives $N E P=1.4 \times 10^{-18} \mathrm{~W} / \mathrm{Hz}^{1 / 2}$ when the device is at $100 \mathrm{mK}$. Whereas the $N E P$ at $357 \mathrm{mK}$ is in good agreement with $N E P_{\text {TEF }}=6.3 \times 10^{-18} \mathrm{~W} / \mathrm{Hz}^{1 / 2}$ determined for the same device from $G_{e-p h}$ near $T_{C}$, it was hard at this time to say to what electron temperature the optical $N E P$ at $100 \mathrm{mK}$ should be attributed. Superconducting transition in magnetic field was rather wide and a full solution of the non-linear heat balance equation will need to be done in order to make an accurate comparison with the electrical $N E P_{T E F}$. The broad transition was likely the reason for the output noise at $100 \mathrm{mK}$ to be much smaller than that at 357 $\mathrm{mK}$.

From the ratio of $N E P$ to $N E P_{T E F}$ at $357 \mathrm{mK}$ and from the offset of the IVCs under different optical power levels, the optical coupling efficiency $\eta=(60 \pm 10) \%$ was found. Given the fact that the Si lens did not have any AR coating, the coupling was very good.

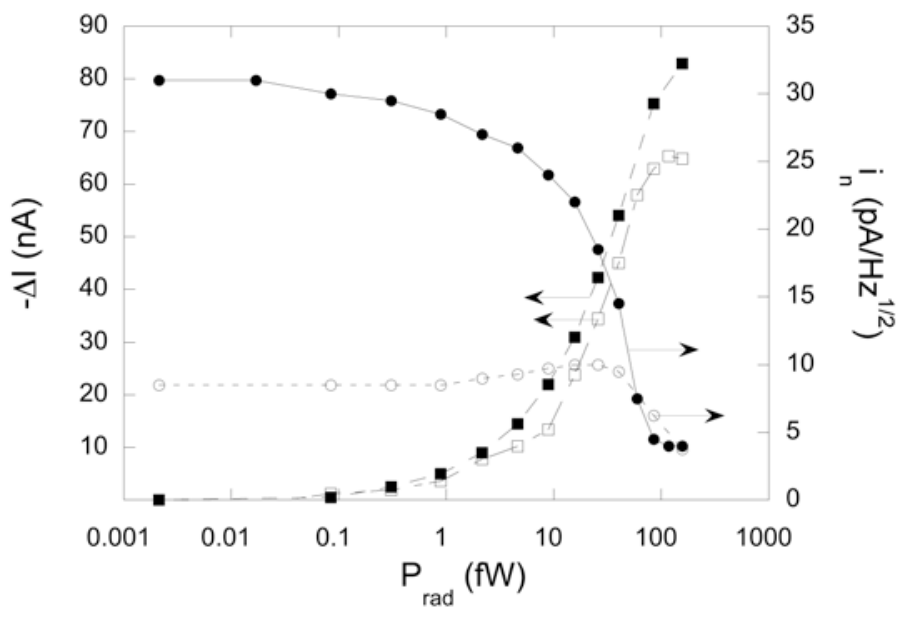

Figure 8. Offset of the bias current caused by $650 \mathrm{GHz}$ radiation and output noise for device \#2. Closed symbols are data at $357 \mathrm{mK}$, open symbols are data at $100 \mathrm{mK}$. The bump in the $i_{n}$ vs $P_{\text {rad }}$ dependence between 10 and $100 \mathrm{fW}$ is due to the detection of photon shot noise.

An interesting feature observed in this experiment was a non-monotonic behavior of the output noise vs $P_{r a d}$ at $100 \mathrm{mK}$. We attribute this to the detection of the fluctuations of radiation power (photon shot noise). Indeed, the noise-like output starts to increase at a few fW of optical power. The corresponding photon shot-noise $N E P_{\text {phot }}=\left(2 P_{\text {rad }} h v\right)^{1 / 2} \sim(1-2) \times 10^{-18}$ $\mathrm{W} / \mathrm{Hz}^{1 / 2}$. Since this is comparable to the detector $N E P$, the fluctuations increase at the output until the detector gets saturated by optical loading and loses its sensitivity. This effect could not be seen at $357 \mathrm{mK}$ since a 100-times greater $P_{\text {rad }} \sim 100 \mathrm{fW}$ is needed to make $N E P_{\text {phot }} \sim 10^{-17} \mathrm{~W} / \mathrm{Hz}^{1 / 2}$. But at such high power the detector is already saturated. At some intermediate temperatures around $200-250 \mathrm{mK}, i_{n}$ remained almost constant with $P_{r a d}$ because of the competition of photon noise detection and saturation effects.

\subsection{Detection of single 8- $\mu \mathrm{m}$ photons}

The small electron heat capacity in the nano-HEB can enable detection of single mid-IR and even far-IR photons with some energy resolution. This may be very useful for the space experiments where the photon flux is so weak that the photons arrive at the detector at a rate smaller than $1 / \tau^{*}$ [7]. It has been predicted [1,7] that the energy resolution in ultimately small submicron-size nano-HEBs can be sufficient for detection of $1 \mathrm{THz}$ photons at $300 \mathrm{mK}$ with extremely low dark count rate. Larger devices (similar to devices \#1 and \#2) have been recently studied using a microwave technique substituting the real photons with equivalent short pulses of $20 \mathrm{GHz}$ radiation ("fauxtons") which were easy to generate and to efficiently coupled to the nao-HEB [8].

Here we report on the first detection of real 8- $\mu \mathrm{m}$ single-photons using device \#1 operating at 50-200 mK. A pulsed QCL generated short (down to $100 \mathrm{~ns}$ ) optical pulses whose energy could be varied by changing the driving current amplitude and the pulse duration. The latter always remained much smaller that the detector time constant in order to 
eliminated the pulse jitter and the pileup of the responses occurring at different instants. The lightpipe loss was adjusted to allow for a convenient pulse energy tuning range so the average number of detected photons per pulse was always less than 1 . The device did not have any antenna or other means to couple to the radiation efficiently so the optical coupling was very poor. Typically, about $10^{4}$ events were recorded and the amplitudes were distributed into 100 bins. The total collection time was limited only by the average laser power, which could cause a heating of the entire mixing chamber if the duty cycle was too high.

Figure 9 shows the photon count statistics at $100 \mathrm{mK}$. The dark counts follow the Gaussian distribution and it has been verified that it is determined by an integral of detector noise over its spectral span. The grey vertical bars show the normalized to unity Poisson distribution $f(n, \mu)=\mu^{n} e^{-\mu} / n$ !, where (in our case) $n$ is the number of simultaneously detected photons, $\mu=0.47$ is the average number of photon per pulse. The energy interval between photon number peaks is $h v$ and the output is fairly linear so the energy resolution of the detector was found to be $h v / \Delta \varepsilon_{F W H M}=1.4$. This resolution remains unchanged from $50 \mathrm{mK}$ to $150 \mathrm{mK}$ and then degrades at $\sim 200 \mathrm{mK}$ completely disappearing near $T_{C}$. In the previous work [8] we determined the minimal resolved energy $\Delta \varepsilon_{F W H M} / h=23 \mathrm{THz}$ at $300 \mathrm{mK}$, which was close to so the called "thermodynamic limit" $\sim \sqrt{k_{B} C_{e} T_{C}^{2}}$. Device \#1 has similar volume and slightly higher $T_{C}$ but nevertheless the energy resolution is noticeably worse. There are several possible causes leading to degradation of $\Delta \varepsilon_{F W H M}$ and they are being currently investigated. One of the causes is apparent from Fig. 9 where the center of the peak corresponding to 0 photon number shifts up with laser power. It means that there are some low-energy excitations (either out-of-band photons emitted by the laser, or secondary photons downconverted in the substrate or electrodes, or non-thermal phonons) contributing to the count statistics. This might possibly lead to the widening of the photon number peaks.

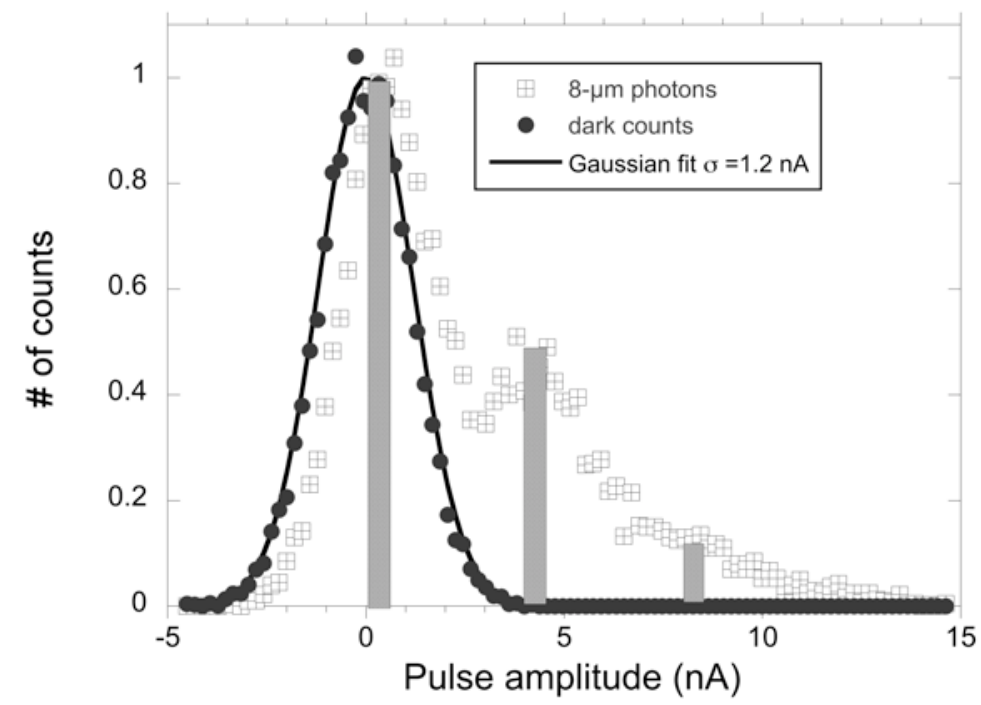

Figure 9. Poisson statistics of single $8-\mu \mathrm{m}$ photon counts for device \#1 at $100 \mathrm{mK}$.

\section{MSQUID MULTIPLEXED READOUT}

The short time constant in nano-HEBs devices $(\sim \mu$ s at 300-400 $\mathrm{mK})$ makes time domain or audio-frequency domain multiplexing impractical. Recently, we have demonstrated the MSQUID approach using dc SQUIDs coupled to X-band microresonators which are, in turn, coupled to a transmission line [13]. We used a 4-element array of devices \#3 operated at $415 \mathrm{mK}$ in a $\mathrm{He} 3$ dewar with an optical fiber access. The microwave signal exhibited $10-\mathrm{MHz}$ wide resonances at individual MSQUD frequencies between $9 \mathrm{GHz}$ and $10 \mathrm{GHz}$. The resonance depth was modulated by the current through the bolometer via a change of the SQUID flux state. The transmitted signal was amplified by a low-noise cryogenic amplifier and downconverted to baseband using an IQ mixer. A 1-dB per $\Phi_{0} / 2$ responsivity was sufficient for keeping the system noise at the level of $\sim 2 \mathrm{pA} / \mathrm{Hz}^{1 / 2}$, which was more than an order of magnitude smaller than the phonon noise in the used HEB devices. The readout bandwidth was limited by the $L_{S} / R$ low-pass filtering at the SQUID input inductance $L_{S}$ that, depending on the bias point, would achieve 0.2-1 MHz. This is sufficiently high for any application temperature. As a matter of fact, the devices were able to detect single near-IR photons $(1550 \mathrm{~nm})$ with a 
time constant of $3.5 \mu \mathrm{s}$. The on-going development work builds a small array of antenna-coupled nano-HEBs using devices \#2. The new generation of MSQUIDs uses lumped element inductors and much smaller separation between the frequency tones $(\sim 5 \mathrm{MHz})$. The system also features the fully digital generation and channelizing of the frequency comb required for the FDM (see Fig. 10). GHz AD and DA converters are fairly common now so it is fair to say that a 1000 pixel array can be multiplexed using this approach in the near future.

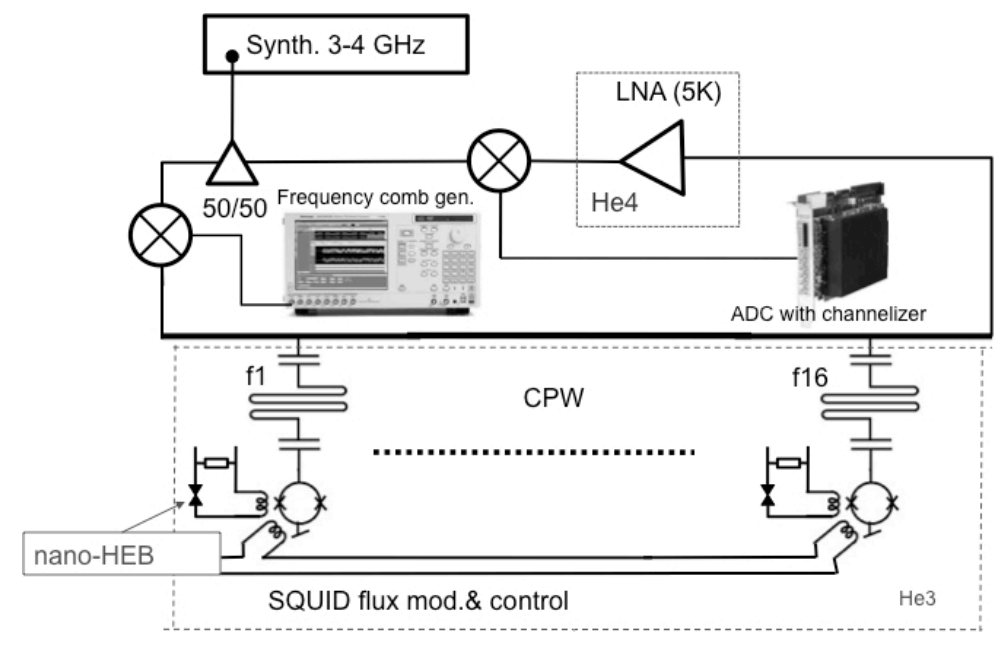

Figure 10. Schematic of the MSQUID based FDM readout for nano-HEB array.

\section{CONCLUSION}

In conclusion, a significant progress has been made in the development of the nano-HEBs based detector technology resulting in the first optical demonstration of the state-of-the-art detector sensitivity in far-IR. The current generation of the detectors together with the MSQUID readout is suitable for building a detector array with $N E P \sim 10^{-17} \mathrm{~W} / \mathrm{Hz}^{1 / 2}$ operating in a He3 dewar. Such a system can be used for photometry, polarimetry, or CMB studies from balloons or SOFIA. The potential for use of nano-HEBs in space for low-background spectroscopy is also very high.

The future R\&D work will address the metrology needed for getting optical $N E P$ down to $10^{-20} \mathrm{~W} / \mathrm{Hz}{ }^{1 / 2}$ as well as the controllable $T_{C}$ reduction in the Ti film or in ready devices using ion implantation of $\mathrm{Mn}$ ions. Another technological step is to adapt the current fabrication process for getting good quality submicron nano-HEBs. This should enable singlephoton detection in mid- and far-IR. This is the whole new arena with major applications in space borne spectrometers on the telescopes with cryogenically cooled primaries. Here the goal is to use single-photon detection in order to achieve $N E P \sim 10^{-20} \mathrm{~W} / \mathrm{Hz}^{1 / 2}$ without going to $50 \mathrm{mK}$ and to increase the dynamic range to $\sim 40-50 \mathrm{~dB}$ and also to implement spectroscopy via calorimetry with resolution $\sim 50$ at $\lambda=8-12 \mu \mathrm{m}$ for exoplanet atmosphere spectroscopy applications. The work in this spectral range will include mid-IR planar antenna development needed for improvement of the coupling efficiency and for reduction of spurious photon downconvertion effects.

\section{ACKNOWLEDGMENT}

This research was carried out at the Jet Propulsion Laboratory, California Institute of Technology, under a contract with the National Aeronautics and Space Administration and funded in part through the internal Research and Technology Development program. The research of S.V. Pereverzev was supported by an appointment to the NASA Postdoctoral Program at the Jet Propulsion Laboratory, California Institute of Technology, administered by Oak Ridge Associated Universities through a contract with NASA.

The authors thank A. Soibel (JPL) for lending the 8- $\mu \mathrm{m}$ QCL and M. Wanke (Sandia National Laboratories) for lending the 100- $\mu \mathrm{m}$ QCL. 


\section{REFERENCES}

[1] Wei, J., Olaya, D., Karasik, B.S., Pereverzev, S.V., Sergeev, A.V. and Gershenson, M.E., "Ultra-sensitive hotelectron nanobolometers for terahertz astrophysics," Nature Nanotechnol. 3, 496-500 (2008).

[2] Karasik, B.S., Pereverzev, S.V., Olaya, D., Wei, J., Gershenson, M.E. and Sergeev, A.V., "Noise Measurements in Hot-Electron Titanium Nanobolometers," IEEE Trans. Appl. Supercond. 19(3), 532-535 (2009).

[3] Kenyon, M., Day, P.K., Bradford, C.M., Bock, J.J. and Leduc, H.G., "Progress on background-limited membraneisolated TES bolometers for far-IR/submillimeter spectroscopy," Proc. SPIE 6275, 627508 (2006).

[4] Benford, D.J. and Moseley, S.H., "Cryogenic detectors for infrared astronomy: the Single Aperture Far-InfraRed (SAFIR) Observatory,” Nucl. Instr. Meth. Phys. Res. A 520, 379-383 (2004).

[5] Leisawitz, D., "NASA's far-IR/submillimeter roadmap missions: SAFIR and SPECS," Adv. Space Res. 34, 631-636 (2004).

[6] Nakagawa, T., "SPICA: space infrared telescope for cosmology and astrophysics," Adv. Space Res. 34, 645-650 (2004).

[7] Karasik. B.S. and Sergeev, A.V., "THz Hot-Electron Photon Counter," IEEE Trans. Appl. Supercond. 15(2), Pt.1, 618-621 (2005).

[8] Santavicca, D.F., Reulet, B., Karasik, B.S., Pereverzev, S.V., Olaya, D, Gershenson, M.E., Frunzio, L. and Prober, D.E., "Energy resolution of terahertz single-photon-sensitive bolometric detectors," Appl. Phys. Lett. 96, 083503 (2010).

[9] Karasik, B.S., Pereverzev, S.V., Olaya, D, Wei, J., Gershenson, M.E. and Sergeev, A.V., "Electrical NEP in hotelectron titanium superconducting bolometers," Proc. SPIE 7020, 70200E (2008).

[10] Mather, J.C., "Bolometer noise: nonequilibrium theory," Appl. Opt. 21, 1125-1129 (1982).

[11] Sergeev, A.V. and Mitin, V., "Electron-phonon interaction in disordered conductors: Static and vibrating scattering potentials," Phys. Rev. B 61, 6041-6047 (2000).

[12] Karasik, B.S., Gaidis, M.C., McGrath, W.R., Bumble, B. and H.G. LeDuc, H.G., "Low Noise in a Diffusion-Cooled Hot-Electron Mixer at 2.5 THz," Appl. Phys. Lett. 71(11), 1567-1569 (1997).

[13] Karasik, B.S., Day, P.K., Kawamura, J.H., Bumble, B. and LeDuc, H.G., "Multiplexing of Hot-Electron Nanobolometers Using Microwave SQUIDs,” AIP Conf. Proc. 1185, 257-260 (2009). 